\title{
MASERS AND THE COSMIC DISTANCE SCALE
}

\author{
Mark J. Reid, James M. Moran, and Carl R. Gwinn \\ Harvard-Smithsonian Center for Astrophysics \\ 60 Garden Street \\ Cambridge, Massachusetts 02138
}

\begin{abstract}
Studies of $\mathrm{H}_{2} \mathrm{O}$ masers have demonstrated the power of VLBI techniques to measure relative positions with sufficient accuracy $(\sim 10 \mu$ as) to determine proper motions and to estimate distances to maser sources throughout the Galaxy. The distance to four $\mathrm{H}_{2} \mathrm{O}$ masers have been determined, and the distance to the center of the Galaxy has been determined to be $7.1 \pm 1.5 \mathrm{kpc}$ from observations of the $\mathrm{H}_{2} \mathrm{O}$ masers in Sgr-B2. Proper motion distances for other $\mathrm{H}_{2} \mathrm{O}$ masers, and possibly for $\mathrm{OH}$ masers, may allow the determination of the fundamental parameters describing the size $\left(R_{o}\right)$ and rotation rate $\left(\Theta_{o}\right)$ of the Galaxy with accuracies of better than $10 \%$. Finally, the measurement of the proper motions of $\mathrm{H}_{2} \mathrm{O}$ masers in nearby galaxies $(<10 \mathrm{Mpc})$ is feasible and offers the possibility of direct calibration of the extragalactic distance scale.
\end{abstract}

\section{INTRODUCTION}

The combination of proper motion data and information about motion along the line-of-sight can be used to determine the distance to a source, as well as to understand the dynamics of the emitting material. The proper motion of a component, $\dot{\theta}_{a p p}$, is given by

$$
\dot{\theta}_{a p p}=\frac{1}{d}\left(\frac{v \sin \theta}{1-\beta \cos \theta}\right),
$$

or in the nonrelativistic limit by

$$
\dot{\theta}_{a p p}=\frac{v \sin \theta}{d},
$$

where $v$ is the speed of the emitting material moving at an angle $\theta$ to the line-ofsight, $\beta=v / c, c$ is the speed of light, and $d$ is the distance to the source. Line-ofsight velocities can be determined indirectly from intensity information, provided bulk relativistic motions are involved, via the equation

$$
I_{a p p}\left(\nu_{a p p}\right)=\underset{169}{D^{3} I_{e m}\left(\nu_{e m}\right),}
$$

M. J. Reid and J. M. Moran (eds.), The Impact of VLBI on Astrophysics and Geophysics, 169-174.

(C) 1988 by the IAU. 
where $D=\sqrt{1-\beta^{2}} /(1-\beta \cos \theta)$, or directly from Doppler (or radial) velocities, $v_{R}$, of spectral lines via

$$
v_{R} \approx v \cos \theta
$$

in the nonrelativistic limit.

Equations (1) and (2) relate measurable quantities to three physical parameters: the distance to the source $(d)$ and the speed $(v)$ and direction $(\theta)$ of the emitting material. VLBI observations of quasars and active galactic nuclei are often interpreted in the context of equations (1a) and (2a), and, assuming $d$ from the source redshift, one can, in principle, determine the "jet" flow speed and direction. Alternatively, for molecular maser sources, equations (1b) and (2b) are appropriate. If proper motions and Doppler velocities are measured for a number of maser spots in one source, one can solve for the parameters by modelling the source dynamics or by assuming an isotropy constraint (e.g., $\left\langle\sin ^{2} \theta\right\rangle=\left\langle\cos ^{2} \theta\right\rangle$ as for a statistical parallax). Both techniques have been used to determine distances to $\mathrm{H}_{2} \mathrm{O}$ maser sources.

\section{MEASURED DISTANCES TO $\mathrm{H}_{2} \mathrm{O}$ MASERS}

Maser emission occurs in the envelopes of newly formed massive stars (interstellar masers) and evolved Red Giant stars (stellar masers). $\mathrm{H}_{2} \mathrm{O}$ and $\mathrm{OH}$ molecules are trace constitutent of these envelopes, and population inversion of the molecular energy levels leads to the appearance of "spots" of emission $\sim 10^{13} \mathrm{~cm}$ is size with brightness temperatures as high as $10^{15} \mathrm{~K}$. Because of the small size and high brightness of interstellar maser spots, they can be used to make precise astrometric measurements that allow proper motions to be determined. With VLBI techniques, relative positions across fields of $\sim 3^{\prime \prime}$ can be measured with an accuracy of $\sim 10$ microarcsec ( $\mu$ as). This accuracy is sufficient to determine proper motions and estimate distances throughout the Galaxy.

Genzel et al. (1981a) measured proper motions of $\mathrm{H}_{2} \mathrm{O}$ maser spots in the Orion-KL source, and found that the spots are expanding about a central point near the massive young star IRc2. Using the two dimensions of spatial information (i.e., relative positions), and the three dimensions of velocity information (i.e., radial and proper motions) available for the maser spots, they constructed a three dimensional kinematic model of the source. The distance of the entire source, which is needed to relate the angular (proper) motions to true velocities, was then estimated by least-squares fitting techniques to be $480 \pm 80 \mathrm{pc}$, in accord with optical luminosity distances of associated O-stars.

Genzel et al. (1981b) and Schneps et al. (1981) measured proper motions of $\mathrm{H}_{2} \mathrm{O}$ masers in $\mathrm{W} 51(\mathrm{M})$ and $\mathrm{W} 51(\mathrm{~N})$, respectively. The motions in these sources appeared largely random, and the technique of statistical parallax was employed to determine distances to the sources. Distance estimates are given by the ratio of the dispersion in Doppler velocities to the dispersion in (each dimension) of the proper motions. The distance to the W51 sources was estimated to be $7 \pm 1.5 \mathrm{kpc}$. This value is consistent with the far kinematic distance to the W51 region.

Recently completed studies of the water masers in the galactic center region, Sgr-B2 (Reid et al. 1988), indicate a phenomenon similar to the Orion case. The $\mathrm{H}_{2} \mathrm{O}$ maser spectrum of Sgr-B2(North) is composed of $\sim 100$ maser spots with an LSR velocity range from 15 to $115 \mathrm{~km} \mathrm{~s}^{-1}$. Spectral-line VLBI maps made from observations between 1980.9 to $\mathbf{1 9 8 2 . 5}$ clearly show up to 50 spots stronger than 
$1 \mathrm{Jy}$ at each epoch. With four telescopes spanning the United States, a synthesized beam of $2.4 \times 0.4$ milliarcseconds (mas) is obtained.

The maser spots in Sgr-B2(North) were slightly resolved and the location of peak brightness was estimated by fitting an elliptical Gaussian model. Formally, the center of a Gaussian brightness distribution can be estimated with a one-sigma precision given by $\sigma_{\theta} \approx 0.5 \theta_{a p p} / S N R$, where $\theta_{a p p}$ is the apparent size (FWHM) of the maser spot (dominated by the synthesized beam) and $S N R$ is the peak signalto-noise ratio for the spot in the map. Plots of position versus time for features at the same line-of-sight velocity were made. These plots indicated that the maser spots typically appeared to move in straight lines on the sky to within a factor of two of the expected (theoretical) position uncertainties.

The dominant motion of the maser spots in Sgr-B2(North) appeared to be expansion. A least-squares fit of a uniformly expanding spherical source resulted in an estimate of the distance to the source of $7.1 \pm 1.5 \mathrm{kpc}$. The estimated expansion velocity is about $45 \mathrm{~km} \mathrm{~s}^{-1}$ and the velocity residuals were about $15 \mathrm{~km} \mathrm{~s}^{-1}$. These residuals are much greater than the measurement errors, suggesting that the source contains "turbulent" motions of about $15 \mathrm{~km} \mathrm{~s}^{-1}$.

Sgr-B2 is almost certainly within $0.3 \mathrm{kpc}$ of the galactic center. Thus, the SgrB2 distance estimate can be used directly as an estimate of $R_{o}$, the distance between the Sun and the galactic center. The $\mathrm{H}_{2} \mathrm{O}$ maser distance estimate of $7.1 \mathrm{kpc}$ to the center of the Galaxy is significantly lower than the previous standard value of $10 \mathrm{kpc}$ and in better agreement with the newly revised IAU value of $8.5 \mathrm{kpc}$ (Kerr and Lynden-Bell 1986).

A decrease in the value of $R_{o}$ has widespread impact on astrophysics. The following is a brief list of items that would be affected by reducing $R_{o}$ :

1) decrease all kinematic distances;

2) reduce the mass of the Galaxy and of the galactic center;

3 ) decrease some extragalactic distances (i.e., increase $H_{0}$ ) by a) favoring recent revisions of the absolute magnitudes of RR Lyrae variables (Frenk and White 1982), which in turn affects distances in the Local Cluster, and b) bringing spiral galaxies closer to match Milky Way size (e.g., DeVaucouleurs 1983);

4) decrease the luminous mass in the Milky Way and increase the "dark matter" in the Local Group (see Trimble 1986); and

5) reduce the total luminosity of $\mathrm{X}$-ray bursts, some of which appear superEddington for $R_{o}=10 \mathrm{kpc}$ (see Ebisuzaki et al. 1984).

\section{FUTURE PROSPECTS FOR GALACTIC DISTANCE MEASUREMENTS}

Analysis of existing data as well as future observations hold the promise of greatly increasing the accuracy of estimates not only of $R_{o}$, but also of the Galactic rotation parameter, $\Theta_{o}$. If one assumes circular rotation in the Galaxy, then the observed radial velocity, $v_{R}$, and absolute source proper motion, $\mu_{\ell}$, are given by

$$
v_{R}=R_{0} \sin \ell\left(\frac{\Theta}{R}-\frac{\Theta_{0}}{R_{0}}\right)
$$

and

$$
\mu_{\ell}=\frac{\Theta}{d}\left[\left(\frac{R_{0}}{R}-1\right) \cos \ell-\frac{d}{R}\right]
$$


where $R$ and $\Theta$ are the distance and rotation speed of the source with respect to the galactic center, $d$ is the distance of the source from the Sun, and $\ell$ is the galactic longitude of the source. After some algebraic manipulation (and with the assumption of a flat rotation curve), one can use measurements of $d, v_{R}$, and $\mu_{\ell}$ to give estimates of $R_{0}$ and $\Theta_{0}$ as follows:

$$
R_{0}=\frac{\rho^{2}-d^{2}}{2(\rho-d \cos \ell)}
$$

and

$$
\Theta_{0}=-\frac{v_{R}}{\sin \ell}\left[\frac{\rho^{2}-2 \rho d \cos \ell+d^{2}}{2 \rho(\rho-d \cos \ell)}\right]
$$

where

$$
\rho=R_{0}-R=\left(\frac{\cos \ell}{d}-\frac{\mu_{\ell} \sin \ell}{v_{R}}\right)^{-1} .
$$

From these equations one can see that, in general, measurement of the absolute proper motion as well as the source distance are needed to determine $R_{0}$ and $\Theta_{0}$ with data from a single source. A proper motion experiment to measure the motion of a source against the extragalactic background, such as that of Backer and Sramek (1982) for Sgr $\mathrm{A}^{*}$, is possible using $\mathrm{H}_{2} \mathrm{O}$ and/or $\mathrm{OH}$ masers. Analysis of equations (5) and (6), however, indicates that only sources near the solar circle (and not too close to the Sun) or sources near the galactic center can be used if deviations from circular motions $\left(\sim 10 \mathrm{~km} \mathrm{~s}^{-1}\right)$ and errors in distances $(\approx 10 \%)$ are not to affect the estimates of $R_{o}$ and $\Theta_{o}$ too seriously.

Note that for a source on the solar circle, such as W49, the results just derived have relavence for distances estimated from internal motions, even if the absolute proper motion of the source is not measured. Near the solar circle, the terms involving $\rho\left(=R_{0}-R \approx 0\right)$ in equations (5) and (6) can be much smaller than $d$. This results in an estimate of $R_{0}$ dependent only on the measurement of $d$. In this case, equation (5) becomes

$$
R_{0} \approx \frac{d}{2 \cos \ell}
$$

independent of the measurement of the absolute proper motion $\mu_{\ell}$. The formal uncertainty in estimating $R_{o}, \sigma_{R_{0}}$, is given by

$$
\frac{\sigma_{R_{0}}}{R_{0}} \approx \frac{\sigma_{d}}{d}
$$

Unfortunately, an estimate of $\Theta_{0}$ depends on both $\mu_{\ell}$ and $d$, and near the solar circle, equation (6) reduces to

$$
\Theta_{0} \approx-\frac{d \mu_{\ell}}{2 \cos \ell}
$$

The most luminous maser in the Galaxy, W49, is known to be on the far side of the galactic center (from hydrogen absorption studies). It has a low radial velocity and, therefore, is nearly on the solar circle. Thus, $R_{o}$ can be accurately determined from the distance to $W 49$, even assuming a crude value for $\Theta_{o}$. For $\ell_{W 49}=43^{\circ}$ and 
$d_{W 49}=12 \mathrm{kpc}$, circular rotation (with $R_{0}=8.5 \mathrm{kpc}$ and $\Theta_{0}=220 \mathrm{~km} \mathrm{~s}^{-1} \mathrm{pc}^{-1}$ ) gives $v_{R}=6 \mathrm{~km} \mathrm{~s}^{-1}$ and $\mu_{\ell}=-5.4$ mas $\mathrm{yr}^{-1}$. By measuring $v_{R}$ and $\mu_{\ell}$ exactly and $d$ with an error of $1.2 \mathrm{kpc}(10 \%), R_{0}$ and $\Theta_{0}$ are estimated with uncertainties of $0.8 \mathrm{kpc}$ and and $20 \mathrm{~km} \mathrm{~s}^{-1}$, respectively. If we add "noise" to the motion of the source in the amount of $10 \mathrm{~km} \mathrm{~s}^{-1}$ to simulate deviations from circular motion or measurement error in $\mu_{\ell}$, the uncertainties for $R_{o}$ and $\Theta_{0}$ are increased only slightly to $1.0 \mathrm{kpc}$ and $23 \mathrm{~km} \mathrm{~s}^{-1}$, respectively.

\section{EXTRAGALACTIC DISTANCES}

$\mathrm{H}_{2} \mathrm{O}$ masers have recently been discovered in about a dozen galaxies. For example, several sites of maser activity are known in M33, a galaxy about $0.7 \mathrm{Mpc}$ from the Sun. The strongest $\mathrm{H}_{2} \mathrm{O}$ maser in $\mathrm{M} 33$ has a flux density of about $5 \mathrm{Jy}$. The most distant extragalactic $\mathrm{H}_{2} \mathrm{O}$ maser known is in the nucleus of the active galaxy NGC 3079. This maser reaches a peak flux density of about $10 \mathrm{Jy}$; at the distance of $16 \mathrm{Mpc}$ this maser is emitting $500 L_{\odot}$ in the $\mathrm{H}_{2} \mathrm{O}$ line, assuming istropic emission. Detections of VLBI fringes have been reported from the galaxies NGC 3079 by Haschick et al. (1987) and from NGC 4258 by Claussen et al. (1987).

Two types of proper motion studies can be done to estimate distances to galaxies: 1) measurement of the internal motions within one source (as done for example for Orion, W51 and Sgr-B2), and 2) measurement of the orbital motion of an entire source around the galaxy. Regarding the first type of study, galactic $\mathrm{H}_{2} \mathrm{O}$ maser sources have internal motions with a dispersion of about $30 \mathrm{~km} \mathrm{~s}^{-1}$. At a distance of $1 \mathrm{Mpc}$, such motions correspond to transverse angular velocities of $6 \mu \mathrm{as} \mathrm{yr}^{-1}$. The second type of proper motion study involves measuring orbital motions due to galactic rotation of $\mathrm{H}_{2} \mathrm{O}$ masers with respect to a "reference" source, e.g., an $\mathrm{H}_{2} \mathrm{O}$ maser or continuum source located elsewhere in the galaxy (perhaps at the center). In this latter case, the relative velocities are $\sim 300 \mathrm{~km} \mathrm{~s}^{-1}-$ an order of magnitude greater than internal source motions. If the orbital speed (rotation curve) and inclination of the galaxy are known, then measurement of the proper motion directly yields the galaxy's distance. A maser located away from the center of a face-on galaxy $1 \mathrm{Mpc}$ from the Sun moves $60 \mu \mathrm{as} \mathrm{yr}^{-1}$, a motion that over one year is a significant fraction of the synthesized beam of intercontinental VLBI arrays and comparable to the synthesized beam of proposed space-based VLBI observations.

The most crucial aspect in the measurement of proper motions of $\mathrm{H}_{2} \mathrm{O}$ masers is to locate reliably each maser spot at several epochs. Maser spots often cluster together. For example, the strongest maser source in the Milky Way is in W49 $(d \approx 12 \mathrm{kpc})$. This source contains about 100 maser spots stronger than $100 \mathrm{Jy}$, with large numbers of these maser spots separated by less than 0.03 arcsec. At a distance of $1 \mathrm{Mpc}$, this separation would be about 0.3 mas, comparable to the best resolution obtainable by an Earth-based array. Once two or more maser spots with similar radial velocities fall within the interferometer beam, intensity variations mimic position shifts. This leads to a "confusion" limit. For example, an Earthbased array observing a source like W49 in a galaxy $1 \mathrm{Mpc}$ distant would not be able to determine the proper motions of the clustered spots. The higher angular resolution provided by future space-based VLBI observations may prove crucial in resolving the structure of $\mathrm{H}_{2} \mathrm{O}$ maser clusters, thereby removing the "confusion" problem (as well as allowing motions to be determined over a shorter time base). 
Even now, measurements of extragalactic proper motions are feasible for known sources with the largest existing radio telescopes and reasonable integration times under good weather conditions. However, we note that no nearby galaxy has yet been searched completely for $\mathrm{H}_{2} \mathrm{O}$ masers. A major program to find the best candidate galaxies for proper motion studies still needs to be done. Since $\mathrm{H}_{2} \mathrm{O}$ proper motions yield a direct estimate of the distance to a source, independent of absolute luminosities and extinctions, they share none of the sources of systematic error usually associated with distance estimates. Thus, obtaining a distance to even one galaxy would provide a crucial check on the calibration of Cepheids and of other distance indicators, and hence could remove significant sources of error in the determination of the Hubble constant.

\section{REFERENCES}

Backer, D. C., and Sramek, R. A. 1982, Ap. J., 260, 512.

Claussen, M., et al. 1987, this volume.

DeVaucouleurs, G. 1983, Ap. J., 268, 451.

Ebisuzaki, T., Hanawa, T., and Sugimoto, D. 1984, P.A.S. Japan, 36, 551.

Frenk, C. S., and White, S. D. M. 1982, M. N. R. A. S., 198, 173.

Genzel, R., Reid, M. J., Moran, J. M., and Downes, D. 1981a, Ap. J., 244, 884.

Genzel, R., et al. 1981b, Ap. J., 247, 1039.

Haschick, A. D., et al. 1987, this volume.

Kerr, F. J., and Lynden-Bell, D. 1986, M. N. R. A. S., 221, 1023.

Reid, M. J., Schneps, M. H., Moran, J. M., Gwinn, C. R., Genzel, R., Downes, D., and Ronnang, B. 1988, Ap. J., in press.

Schneps, M. H., Lane, A. P., Downes, D., Moran, J. M., Genzel, R., and Reid, M. J. 1981, Ap. J., 249, 124.

Trimble 1986, Comments Astrophys., 11(5), p. 257. 